\title{
Decoupling the molecular regulation of perenniality and flowering in bulbous barley (Hordeum bulbosum)
}

\author{
Dana Fuerst ${ }^{1}$, Bar Shermeister ${ }^{1}$, Tali Mandel ${ }^{1}$, Sariel Hübner ${ }^{1, \#}$ \\ ${ }^{1}$ Galilee Research Institute (MIGAL), Tel-Hai Academic College, Upper Galilee, 11016, Israel. \\ ${ }^{\#}$ Corresponding author
}

\section{ABSTRACT}

Global crop production is being challenged by rapid population growth, declining natural resources, and dramatic climatic turnovers. These challenges have prompted plant breeders to explore new ventures to enhance adaptation and sustainability in crops. One intriguing approach to make agriculture more sustainable is by turning annual systems into perennial which offers many economic and biodiversity-friendly benefits. Previous attempts to develop a perennial cereal crop employed a classical breeding approach and extended over a long period with limited success. Thus, elucidating the genetic basis of perenniality at the molecular level can accelerate the breeding process.

Here, we investigated the genetic basis of bulb formation in the barley congener species Hordeum bulbosum by elucidating the transcripts presence/absence variation compared with other annual species in the Poaceae, and a differential expression analysis of meristem tissues. The PAV analysis recaptured the expected phylogeny and indicated that $H$. bulbosum is enriched with developmental and disease responsive genes that are absent among annual species. Next, the abundance of transcripts was quantified and allowed to identify differentially expressed genes that are associated with bulb formation pathways in addition to major circadian clock genes that regulate flowering. $A$ first model for the bulb formation pathway is suggested and include developmental and starch biosynthesis genes. To the best of our knowledge this is the first transcriptome developed for $H$. bulbosum and the first attempt to describe the regulation of bulb initiation in cereals at the molecular level. 
Keywords: Transcriptome; Perenniality; Barley; Hordeum bulbosum; bulb initiation; flowering time

\section{INTRODUCTION}

It is now recognized that agriculture can be made far more sustainable by transitioning many annual agricultural systems to perennial (Ryan et al., 2018). Perennials are yearround crops harvested multiple times over several seasons, a feature that offers many environmental and economic benefits. Unlike annual crops, perennials are thriftier and improve soil structure and water retention capacity, contribute to increased mitigation practices to cope with climate change, and promote biodiversity and ecosystem functions (Kantar et al., 2016; Lundgren et al., 2020). Among annual crops that are cultivated across over $70 \%$ of global croplands, many staple crops could potentially be transferred to a perennial life habit by hybridization and other genomic engineering techniques (Hübner and Kantar 2021). Generally, there are two main approaches to develop a new perennial crop: de novo domestication of a wild perennial plant, and introgression of perennial traits into an annual crop through hybridization practices (Cui et al., 2018; Kantar et al., 2018). Several attempts to develop a perennial cereal crop were made over the years with little commercial success. For example, efforts to develop perennial wheat varieties through hybridization with wild relatives were successful in generating stable amphiploids (Amstrong, 1936; Larkin et al., 2014), yet grain yield declined quickly under field conditions. Thus, introgression of perenniality into an annual crop is a challenging long endeavor. Targeting crosses and directing selection based on a deep understanding of the molecular regulation of the perenniality traits can potentially accelerate this tedious process (Kantar et al., 2018). Recent developments in biotechnology have prompt new methods to introduce a trait of interest into a crop through genome editing. This technology can be used for de novo domestication of wild species by targeting genes that regulate domestication syndrome traits including seed shattering and awn length (Yu et al., 2021), fruit size and number, 
and the nutritional value (Gasparini et al., 2021). Another approach, considers perenniality as a syndrome which includes a variety of interacting traits, thus understanding the underlying molecular mechanism is necessary to allow breeding efforts to focus on specific components of perenniality (Lundgren and Marais 2020).

Barley (Hordeum vulgare) ranks fourth among cereal crops in cultivated area (www.fao.org/faostat) and is known for its enhanced adaptation to a wide range of environments (Haberer et al., 2015). Very few attempts were made so far to breed for perenniality in barley although there are perennial species in the genus that are crosscompatible with cultivated barley.

Hordeum bulbosum is a wild cereal species that has diverged from barley 4 million years ago and is highly abundant in the Mediterranean region (Brassac et al., 2015). Despite the morphological similarity between barley and $H$. bulbosum, the latter is a perennial species merit to its bulb organ, which allows to pass the dry summer season by entering a dormancy period. In the late fall, when the days are getting shorter, temperature declines and the first rain of the season, the new $H$. bulbosum leaves sprout from regenerating buds that are located at the periphery of the bulb organ. After emergence, the $H$. bulbosum plant continues to grow and establish until the end of December when days are getting longer and signal the plant to shift from vegetative growth to flowering and to form a new bulb organ (Koller et al., 1960). Previous studies in anion (Allium cepa L.) indicated that the transition from short to long days induce bulb formation and development through the regulation of FT homologs and other flowering regulating genes (Lee et al., 2013). However, little is known about the regulation of bulb formation in $\mathrm{H}$. bulbosum and the underlying molecular mechanism remains largely unknown. To address this, advanced genomic infrastructure and proper molecular tools adjusted for $H$. bulbosum are mandatory, yet very few tools were developed so far for this nonmodel species. Recently, a reference draft genome was developed for a haploid $H$. bulbosum, but this resource is highly fragmented and un-annotated, thus the representation of the gene space is partial (Wendler et al., 2017). Another efficient approach to identify genes of interest in a non-model organism is RNA sequencing which 
allows to efficiently develop a reference transcriptome and test for the expression of genes in response to a signal (Costa-Silva et al., 2009). Assembling de novo a

transcriptome for $H$. bulbosum has many benefits and can significantly improve the gene space representation and the identification of candidate genes involved in a perennial organ formation and development.

Here, we describe the assembly of a reference transcriptome for $H$. bulbosum and the analysis of the presence/absence variation and differential gene expression to identify candidate genes that are involved in the regulation of bulb formation. We hypothesize that the development of a bulb organ in $H$. bulbosum is initiated in the shoot meristem during the vegetative stage in response to transition from short-day to long-day regime. We show that bulb formation is regulated by genes and pathways that are coupled with the circadian clock and flowering but the experimental design used allowed to partially deconvolute them. We further explore the set of candidate genes identified and suggest a molecular model for the regulation of bulb initiation in $\mathrm{H}$. bulbosum.

\section{MATERIALS AND METHOD}

\section{Plant material}

A controlled experiment was set from a random batch of seeds sampled at Alon HaGalil in northern Israel $\left(32^{\circ} 45^{\prime} 35.2^{\prime \prime} \mathrm{N} 35^{\circ} 13^{\prime} 30.5^{\prime \prime E}\right)$, from single spikes of $H$. bulbosum and $H$. spontaneum plants. Selected seeds were sown in planting trays, and placed in a cold $\left(4^{\circ} \mathrm{C}\right)$ dark room for 15 days to break dormancy. Germinated seedlings were transferred into a growth-room under short-day regime (SD; 8h:16h light:dark), and constant temperature of $16^{\circ} \mathrm{C}$. After an establishment period of two weeks, seedlings were transferred into 3-liter pots containing a soil mixture (“GREEN 90", Ben-Ari Ltd., Israel) and slow release fertilizer (Osmocote, Everris International B.V. Heerlen, The Netherlands). After nine weeks in growth-room under SD regime, plants of both species were divided randomly between three groups in accordance with the three treatments in the experiment $\left(T_{0}, T_{2}, T_{4}\right)$. Each treatment was set in three biological replicates, thus 
a total of 9 plants of each species (total 18 plants) were included in the experiment. Shoot meristem tissues were sampled from the first group $\left(T_{0}\right)$ before transition to longday (LD) regime and were immediately frozen in liquid nitrogen. After sampling the $T_{0}$ plants, the conditions in the growth-room were shifted to LD regime (16h:8h, light:dark) and shoot meristems were sampled from the $T_{2}$ plants after 24 hours. After additional 96 hours in LD, shoot merisms were sampled from the third group of $T_{4}$ plants.

For the reference transcriptome assembly, three $H$. bulbosum plants were grown under SD for three weeks and then transferred to LD regime until flowering and development of a mature bulb organ. A total of six tissues were sampled for RNA extraction and sequencing including bulb, anthers, stigmas, leaf, embryo and shoot meristem. For embryo extraction, a single seed was soaked in water for two hours to soften the seed and allow easy removal of the embryo. Altogether, twenty-four tissue samples were obtained and immediately frozen in liquid nitrogen and stored in $-80^{\circ} \mathrm{C}$ until extraction of RNA.

\section{RNA extraction, library preparation and sequencing}

Total RNA was extracted from each of the 24 samples $(9 \mathrm{H}$. spontaneum and $15 \mathrm{H}$. bulbosum) using the RNeasy Plant Mini Kit (QIAGEN cat No./ID 74904) following the manufacturer protocol. For embryo RNA extraction, we used $500 \mu \mathrm{l}$ RLT buffer and 10 $\mathrm{l}$ $\beta-M E$, and for the leaf tissue $500 \mu \mathrm{l}$ RLT buffer and $5 \mu \mathrm{l} \beta-\mathrm{ME}$ as recommended by the manufacturer. To confirm the integrity and quality, total RNA was inspected in both NanoDrop 1000 spectrophotometer and agarose gel electrophoresis. An OD 260/280 ratio of 2.0 and RNA concentration of $100 \mathrm{ng} / \mu \mathrm{l}$ were set as a minimum threshold for adequate quality and quantity. Sequencing libraries were constructed and sequenced at the Technion Genome Center (Haifa, Israel). RNA-Seq libraries were prepared using the TruSeq RNA Library Prep Kit v2 (Illumina Inc., USA) following manufacturer instructions and sequenced on six lanes of HiSeq-2500 Illumina machine. To avoid a lane bias in the expression profiling analysis, samples were pooled and the sixth portion of the pool was sequenced on each of the six lanes. The quality of the sequenced reads was inspected with the software FastQC v.0.11.5 (Andrews, 2010) and low quality reads were trimmed 
and adapters were removed using the default parameters in Trimmomatic v.0.32

(Bolger et al., 2014). Finally, cleaned and high-quality reads were re-inspected using FastQC.

\section{Transcriptome assembly and annotation}

To develop a comprehensive and representative transcriptome for $\mathrm{H}$. bulbosum, 15 RNA-Seq libraries obtained from roots, bulb, leaf, floral reproductive tissues (anthers and stigma) and shoot meristems were sequenced on a HiSeq-2500 Illumina machine. The transcriptome was assembled de novo with the Trinity assembler v1.8 (Grabherr et al., 2011) using the reads generated from all $H$. bulbosum tissues. Briefly, Trinity performs a full transcriptome assembly process in three main stages and include partitioning the data, clustering and graphs construction, and tracing paths in the constructed graphs in parallel. At the end of this process, a linear sequence is obtained for each transcript isoform.

To annotate the transcriptome we used the Trinotate pipeline v-3.1.1 (Bryant et al., 2017). Prediction of open reading frame (ORF) in the assembled genes was conducted with TransDecoder v-5.5.0 (http://transdecoder.github.io). Homolog proteins detection was conducted with blastx and blastp against the SwissProt and UniProt (Boeckmann et al., 2005; the UniPort Consortium, 2021) databases and an e-value cut-off of $10^{-5}$. To identify conserved protein domains, trans membranal regions and rRNA genes, the HMMER toolkit v-3.1b2 (Eddy, 1998) was used against PFam database (Finn et al., 2014). Signal peptides were predicted using SignalP (Petersen et al., 2011). To filter potential contamination in the transcriptome, transcripts that were annotated to species outside the Spermatophyta super-division (seeded plants) were excluded from the dataset. To further evaluate the quality of the assembly, cleaned reads from each of the sequenced libraries were aligned back to the filtered transcriptome using bowtie2 v2.3.5.1 (Langmead et al., 2009) and the alignment statistics was calculated with samtools v1.992 (Li et al., 2009). To evaluate the completeness of the transcriptome, a BUSCO analysis was preformed (Simão et al., 2015) using the Poales database and the transcriptome 
mode. Only longest isoform per gene was inspected to minimize the false identification of duplicated genes.

Finally, the obtained transcriptome was compared with the genomes of Hordeum spontaneum (Jayakodi et al., 2020), Trititcum aestivum (Appels et al., 2018), Oryza sativa (Wang et al., 2018), and Brachypodium distachyon (Vogel et al., 2010) and presence absence variation among species was analyzed. We extracted the longest isoform from each gene to avoid the complex comparison between a transcript and the gene model as annotated in different species. The extracted isoform was then searched in the available draft genome of $H$. bulbosum to exclude genes that are specific to the individual that was sequenced and the assembly methodology in each platform. The genes presence/absence among species was determined using blastn where no-hit of a gene was considered as absent.

\section{Differential expression analysis}

To identify candidate genes involved in the regulation of bulb formation in $\mathrm{H}$. bulbosum a differential expression (DE) analysis was performed using the RNA-Seq data generated from shoot meristem tissues before and after transition from SD to LD photoperiod.

Previous studies on bulb physiology and development (Ofir et al., 1974) have shown that the day-length signal is intensified 8-10 weeks after germination, thus plants were kept in SD regime for this period of time. The experiment included three treatment groups: before the transition to LD regime $\left(T_{0}\right), 24$ hours after transition to LD regime $\left(T_{2}\right)$, and 96 hours after the transition to LD $\left(T_{4}\right)$. For each group, three biological replicates were sampled for RNA extraction and sequencing. Obtained data was trimmed and cleaned with Trimmomatic and only high-quality reads were aligned with Bowtie2 to the transcriptome. To quantify the expression profile of genes in each sample, alignment files were processed with RSEM v.1.2.31 (Li et al., 2011), and genes with differentially expressed profile between treatments were detected with the R package DESeq2 v1.22.2 (Love et al., 2014). To correct the high false discovery rate (FDR) due to multiple testing, we used the Benjamini-Hochberg correction with a cutoff of FDR $\leq 5 \%$. No 
filtering threshold was set for the expression fold change $\left(\log _{2}-\mathrm{FC}\right)$ between treatments to avoid the exclusion of mild differences which is common for regulating genes such as transcription factors (Sha'ar-Moshe et al., 2015).

\section{Gene ontology analysis}

Gene ontology (GO) analysis was conducted to identify enriched biological processes among the different tissues that were used to assemble the transcriptome, among genes showing presence/absence variation (PAV), and among genes that were differentially expressed in response to transition from SD and LD regimes. GO terms were extracted from the annotation file generated for the transcriptome and used for enrichment analysis with the package topGO v3.11 (Alexa et al., 2006) in R. The GO analysis was performed with default parameters and statistical significance was determined with Fisher exact test. The $p$-values reported were not adjusted following the recommendations of the package authors. Heatmaps were generated with 'gplot' v3.1.1 (Warnes et al., 2009) for the top significant GOs terms.

\section{RESULTS}

\section{The $H$. bulbosum transcriptome}

A total of 140 billion base-pairs from 15 tissue samples were processed in order to assemble the $H$. bulbosum transcriptome after removing 2-4\% of reads per sample due to unsatisfying quality. The total length of the obtained transcriptome was $650 \mathrm{Mbp}$ comprising of 707,824 transcripts (Table S1). Following the assembly, transcripts were annotated and filtered to include only genes that are present in the Spermatophyte super-division database. This filtering approach allowed to avoid potential contamination while maintaining information from species outside the Poacea that may be relevant for the identification of bulb regulating genes (e.g. onion, garlic). A total of 138,696 transcripts remained after filtering, of which 42,045 are unigenes with a contig N50 of 2,758 bp. Reads from all libraries of $H$. bulbosum were aligned to the filtered transcriptome and the average alignment rate was 59\% (Table S2, Figure S1). 
To evaluate the completeness of the assembled transcriptome, BUSCO analysis was performed using the Poales genes database (4,896 BUSCOs) and the transcriptome mode. Altogether, 3,117 BUSCO genes were detected of which $92 \%$ are complete genes and $84 \%$ are single copy genes. Next, the assembled transcriptome was compared with the available reference draft genome for $\mathrm{H}$. bulbosum using the BUSCO pipeline and the Poales genes database. Expectedly, more genes were detected in the draft genome $(4,119)$ than in the transcriptome because the later was targeted to specific tissues and timing. Interestingly, once unrepresented genes were excluded, the number of complete genes was higher (92\%) and the number of duplicated genes was lower (87\%) in the transcriptome compared with the draft genome (Figure 1, Table S3). These results emphasize the advantage of the transcriptome in representing targeted genes for expression profiling and analysis.

To further explore the transcriptome composition and the expression profile in each tissue (leaf, root, flower organs, bulb and embryo), a subset of 34,282 genes with a minimum coverage of $2 X$ in at least one tissue were extracted. Genes that were expressed only in shoot-meristems were excluded from this analysis due to overrepresentation among sequenced libraries (9 libraries). The number of genes detected in each tissue was quantified and indicated that $43 \%$ of the expressed genes are shared among all 6 tissues, and 19\% are tissue-specific (Figure 1). High rate of tissue-specific genes was observed among embryo $(2,505)$, followed by roots $(1,429)$, anthers $(996)$, bulb (837), leaf (576), and stigma (392), after confirming that the number of genes detected in a tissue is not biased by the amount of RNA extracted $(r=0.29, p=0.57$; Figure S2). To further explore the underlying function of genes that were expressed in different tissues or tissue-specific genes, a gene ontology (GO) enrichment analysis was conducted (Table S4). Among GO categories, 19 were significantly enriched across all tissues and included response to stimulus, developmental process and biological regulation. Within tissues, GO categories that were significantly enriched in a specific tissue included biological processes that are identified with the corresponding tissue (Figure 1C). For example, leaf tissue was enriched with photosynthesis processes 
bioRxiv preprint doi: https://doi.org/10.1101/2021.11.11.468190; this version posted November 12, 2021. The copyright holder for this preprint (which was not certified by peer review) is the author/funder, who has granted bioRxiv a license to display the preprint in perpetuity. It is made available under aCC-BY-NC 4.0 International license.

including light harvest in photosystem-I (GO:0009768, $\left.p=1.70 \times 10^{-5}\right)$ and chlorophyll biosynthesis (GO:0015995, $p=8.17 \times 10^{-3}$ ), embryo tissue was enriched with germination processes (GO:0009415, $\left.p=9.40 \times 10^{-8}\right)$, and phyllotaxis $\left(\mathrm{GO}: 0060772, p=2.30 \times 10^{-6}\right)$. A

list of $\mathrm{GO}$ terms identified in each tissue is provided in the supplementary materials (Table S4).

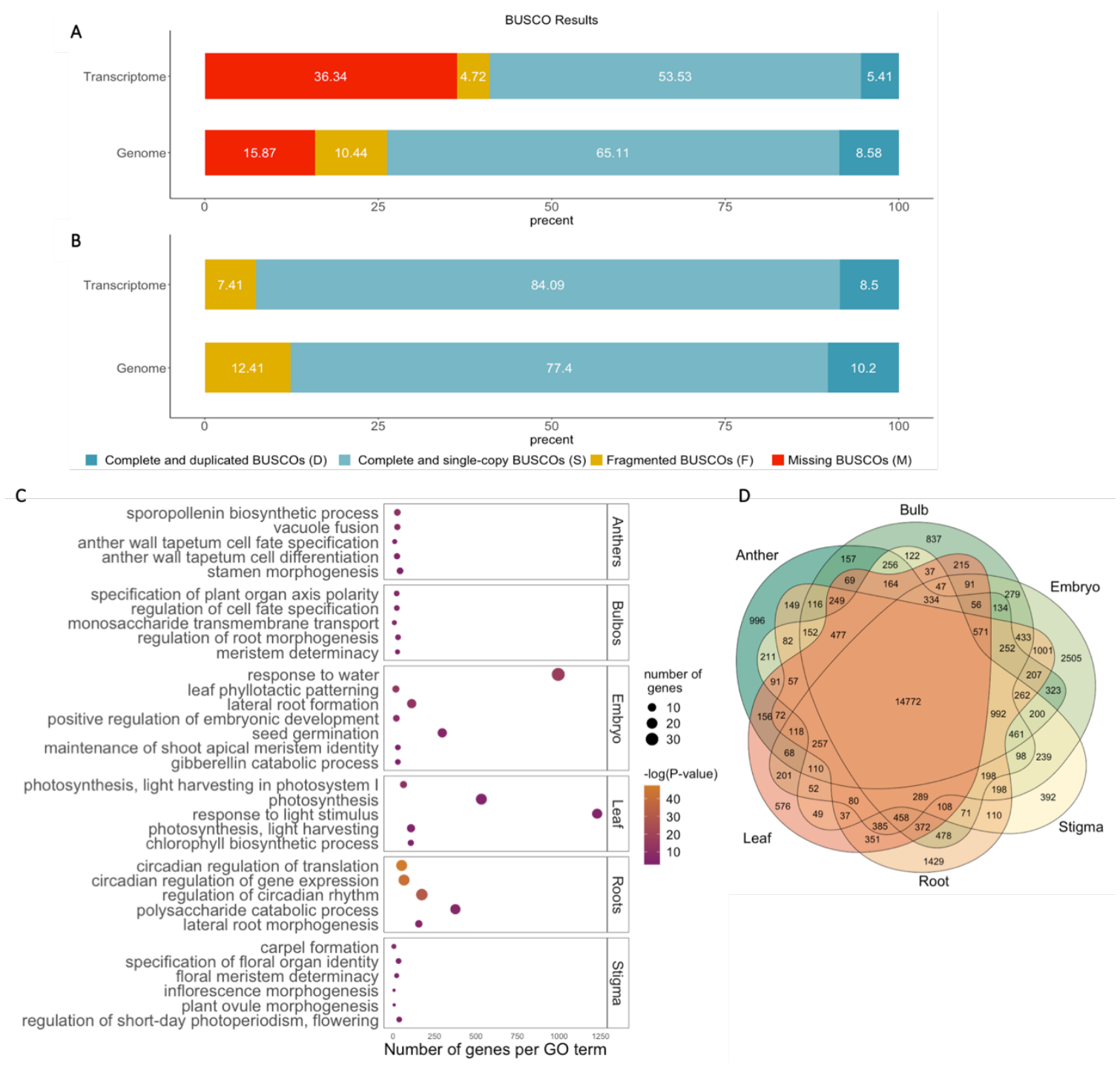

Figure 1. Evaluation and analysis of $H$. bulbosum transcriptome. A,B) Proportion of BUSCOs in the de novo transcriptome assembly compared with the draft genome of $H$. bulbosum including all BUSCO genes (A), and after removing missing BUSCOS (B). C) Significantly enriched GO terms in each tissue that was included in the transcriptome assembly. The circle size is proportional to the number of genes associated with in each GO term. D) Venn diagram of shared and unique genes expressed among tissues. 
The ability to generate a perennial organ among Triticeae species is rare, thus gene gain or loss dynamics may underlie the process of bulb formation and development in $H$. bulbosum. To test this and identify genes that are present in $\mathrm{H}$. bulbosum and absent among annual grass species, the presence/absence variation (PAV) between $H$. bulbosum, $H$. spontaneum, $T$. aestivum, $B$. distachyon and $O$. sativa was investigated. Among the 42,045 assembled genes in the transcriptome, 2,591 were absent from the draft genome and were excluded from the PAV analysis. All remaining transcripts were compared to all other species to identify and quantify the level of homology for each transcript using blastn. A gene was considered absent in a species only if no-hit was obtained. This conservative approach allows to target, with high confidence, genes in $H$. bulbosum that are absent in the other annual species albeit with some level of false negative (undetected genes). A phylogenetic tree constructed from the PAV across all genes supported the expected topology among the Triticeae. The lowest rate of genes shared with $H$. bulbosum was observed for O. sativa (47\% of $H$. bulbosum genes) which has diverged from $H$. bulbosum 50 MYA, and highest rate (91\%) was observed for $H$. spontaneum which has diverged from $H$. bulbosum 4 MYA (Figure 2A,C). Among the 39,454 genes for which PAV was detected, 154 genes were unique to $H$. bulbosum and 626 were present only within the Hordeum genus. Among unique genes to $H$. bulbosum, number of disease resistance genes were detected including RGA-like genes which are involved in plant-pathogen interaction. Among the genes detected in the transcriptome assembly and absent from all genome assemblies (including the $H$. bulbosum draft genome), several organ development genes were detected including the 14-3-3-like gene which promote tuberization in potato (Teo et al., 2017). Interestingly, 14-3-3-like was also significantly differentially expressed in response to the transition to LD regime in $H$. bulbosum but was absent from the annual $H$. spontaneum.

To further investigate the biological process that underlie the detected PAV genes, a GO enrichment analysis was conducted. A total of $111 \mathrm{GO}$ terms were significantly enriched among genes that were detected only in $H$. bulbosum and absent in annual species and included response to biotic stress (GO:0050832, $p=1 \times 10^{-30} ; \mathrm{GO}: 0046718, p=9.9 \times 10^{-3}$ ), 
sugar accumulation, starch metabolism and organ development (GO:0005982, $p=$

$2.8 \times 10^{-2}$; GO: 0042753, $p=7.9 \times 10^{-5} ; \mathrm{GO}: 0010082, p=1.4 \times 10^{-3} ; \mathrm{GO}: 0048367, p=3 \times 10^{-2}$ )

(Figure 2B, Table S5).
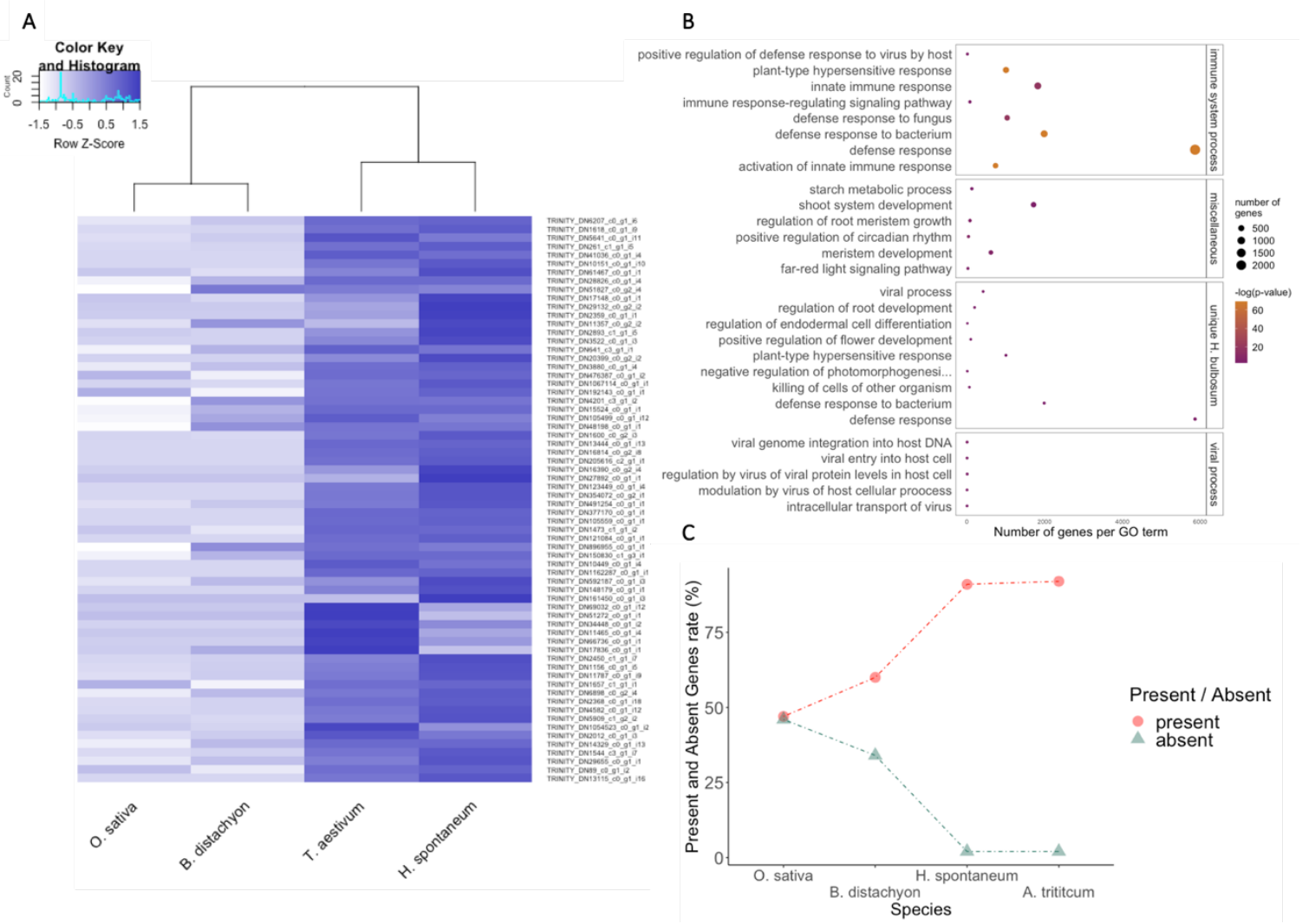

Figure 2. Presence/absence variation (PAV) analysis for $\mathrm{H}$. bulbosum and annual species of the Poaceae. A) Heatmap of PAV genes in $H$. bulbosum compared with $O$. sativa, $B$. distachyon, $H$. spontaneum, and T. aestivum using a normalized bit-score values obtained from blastn analysis. Genes with no-hit were given a score of 0 and darker colors indicate higher homology. B) Significantly enriched GO terms among genes that were identified in $\mathrm{H}$. bulbosum but are absent in at least one species or unique to $H$. bulbosum. C) Percent of P/A genes among different species.

\section{Identification of candidate genes involved in bulb formation}

Previous work suggested that the bulb initiation signal is intensified at the meristem after 8-10 weeks in short-day regime (Ofir et al., 1974). Therefore, meristem tissues were sampled 10 weeks after germination $\left(T_{0}\right)$ and then were transitioned to LD regime. Following the transition, meristem tissues were sampled after $24\left(T_{2}\right)$ and $96\left(T_{4}\right)$ hours under LD and RNA was extracted and sequenced (Figure 3A). An average of 92 million paired-end reads were obtained for each sample and were aligned to the assembled 
transcriptome yielding an average of $60 \%$ and $30 \%$ properly aligned and uniquely mapped reads, respectively (Table S2). The high rate of multi-mapped reads with low mapping quality was further investigated using a subset of 1000 reads that were randomly sampled and searched against the NCBI non-redundant database. The analysis indicated that the high rate of multi-mapped reads is attributed to the large number of duplicated genes in the assembled transcriptome. Finally, a total of 36,929 genes were detected among the meristem tissues with an average coverage of $66 \%$ and an average depth of 38X (Table S6).

Next, the expression profile at different treatments and timepoints was investigated. A PCA conducted for the different samples using the expression profile of all genes confirmed the assignment of samples to treatments (Figure S3). A major challenge in the experiment was that LD signal initiates the formation of a bulb organ, but as in other species, also the transition to flowering. To decouple these two processes and target genes that underlie the regulation of bulb formation, the experiment was conducted also for the annual species $H$. spontaneum following the same experimental setup. We hypothesize that unlike flowering regulating genes that are expected to be detected in both species, bulb formation and development genes will be detected only in the perennial species (H. bulbosum).

Following the transition of $H$. bulbosum from SD to $\operatorname{LD}\left(\mathrm{T}_{0} / \mathrm{T}_{2}\right)$, a total of 1568 significantly differentially expressed genes (DEGs) were detected of which 837 were upregulated and 731 were downregulated (Figure 3). After four days in LD ( $\left.T_{0} / T_{4}\right)$ the effect has strongly declined and only 102 genes were upregulated and 156 were downregulated. In contrast to the expression profile observed in $\mathrm{H}$. bulbosum, the transition from SD to LD regime in $H$. spontaneum had a mild effect after 24 hours and only 67 upregulated and 57 downregulated DEGs were detected. However, after four days in LD the effect was intensified and 221 upregulated and 713 downregulated DEGs were detected. These results indicate that the day length signal is indeed intensified in $H$. bulbosum due to the long period in SD (10 weeks) while in $H$. spontaneum the effect accumulates gradually after the transition to LD. Interestingly, flowering related genes 
were mainly detected in $H$. bulbosum after four days $\left(T_{4}\right)$ in LD similarly to the response observed in $H$. spontaneum, implying that bulb initiation genes responded to the transition to LD regime before flowering regulating genes (Figure 3E).

To further study the functional context of the detected DEGs in H. bulbosum, a GO enrichment analysis was conducted. The number of significantly enriched GO terms was consistent with the declining trend observed in the expression analysis, thus 183 and 46 enriched GO terms were detected for $T_{0} / T_{2}$ and $T_{0} / T_{4}$ respectively. Among the enriched GO terms in the $T_{0} / T_{2}$ and the $T_{0} / T_{4}$ comparisons, 25 terms are associated with flowering (GO:0045595, $p=0.0038 ; \mathrm{GO}: 0009908, p=0.0036)$, response to photoperiod (GO:0048577, $p=0.0186$ ), and bulb formation (GO:0005986, $p=0.0157$ ).

To further explore the genetic basis of bulb initiation and development, a list of DEGs that are associated with significantly enriched GO terms was extracted (Table S7,S8). Generally, three phases are expected in the process of bulb formation: response to the LD signal, morphogenesis and organ development, and accumulation of sugars in the bulb organ. Among DEGs that underlie enriched GO terms, we detected CONSTANS $\left(\operatorname{logFC}=1.48796\right.$, FDR $\left.=4 \times 10^{-4}\right)$, GIGANTEA $\left(\operatorname{logFC}=-1.61\right.$, FDR $\left.=1.49 \times 10^{-7}\right)$, and the pseudo-response regulator $(P R R 5)$ gene $\left(\operatorname{logFC}=-3.09, F D R=1.71 \times 10^{-17}\right)$ which interact in response to day-length and signal transduction. In addition, we detected the morphogenesis and development regulating genes constitutive photomorphogenesis 9 $(\mathrm{COP})(\log F C=0.6096, \mathrm{FDR}=0.001)$ and MADS-box16 $\left(\log F C=4.33, \mathrm{FDR}=2 \times 10^{-4}\right)$, and the fructose accumulation regulating gene SWEET17 $(\operatorname{logFC}=2.11, \mathrm{FDR}=0.005)$.

In $H$. spontaneum, the response to LD was more prominent 96 hours after the transition to LD with 65 and 123 significantly enriched $G O$ terms in the $T_{0} / T_{2}$ and $T_{0} / T_{4}$ respectively. Among the $\mathrm{GO}$ terms detected in the $T_{0} / T_{4}, 13$ are associated with the response to the LD signal and initiation of flowering (Table S9). GO terms that comprise DEGs that were also detected in $H$. bulbosum included MADS-box18, CONSTANS and PRR95, thus these genes are likely associated with initiation of flowering rather bulb formation and development. 

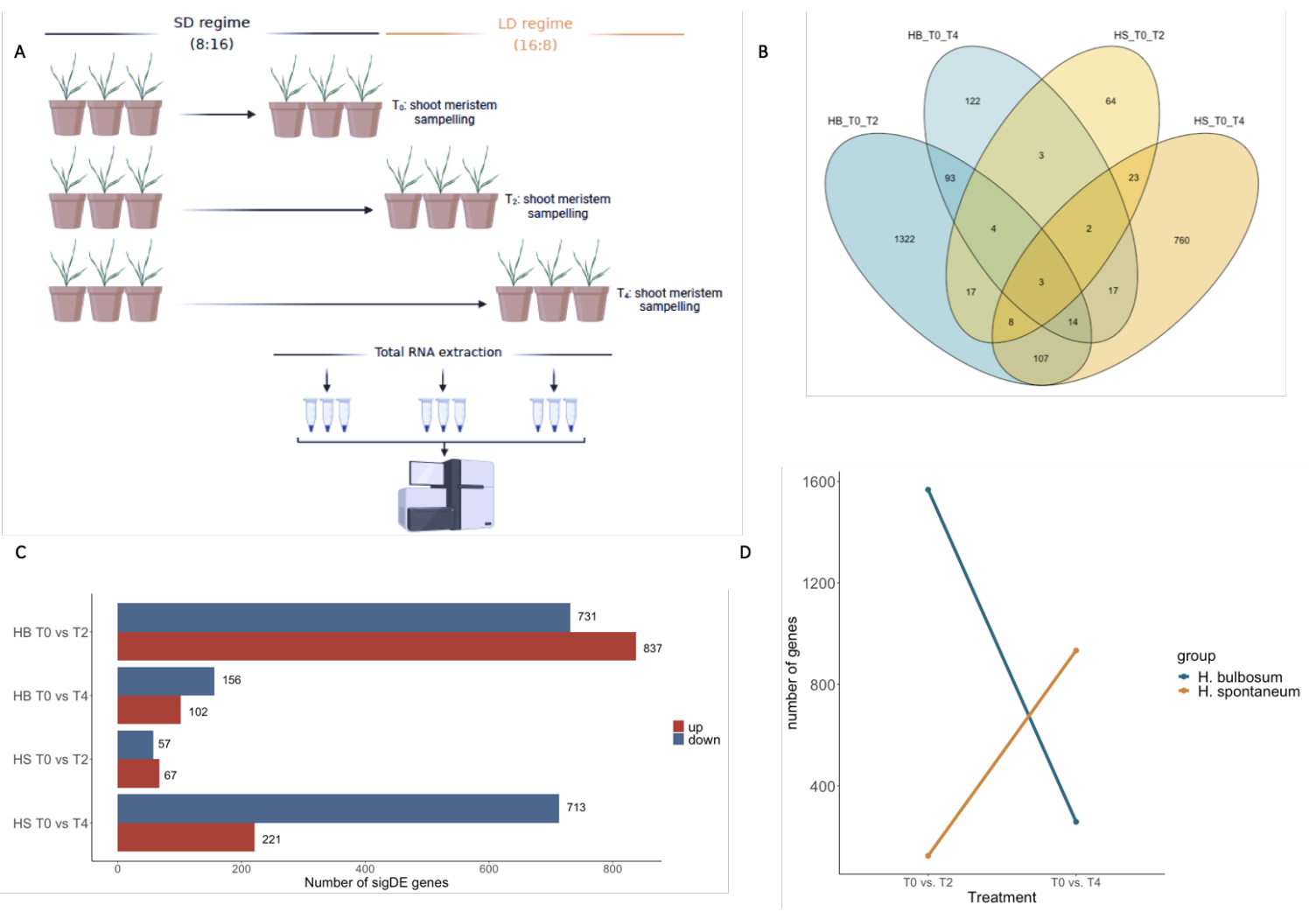

Figure 3. Differential expression analysis of meristem tissues in response to shift in day-length regime. A) A scheme diagram of the experiment. B) A Venn diagram for DEGs detected in $\mathrm{H}$. bulbosum and $\mathrm{H}$. spontaneum. C) Counts of up and down DEGs in H. bulbosum (HB) and H. spontaenum (HS). D) Total number of DEGs in H. bulbosum and $H$. spontaneum along time/treatment.

\section{DISCUSSION}

Despite the many benefits of modern agriculture, the associated environmental impact must be reduced to meet current global efforts to mitigate climate change. Thus, agriculture must adopt a more sustainable strategy to face the rising demand for food while natural rescues are declining. One way to reduce the negative environmental impact of agriculture is to shift crop plants from annual system to a perennial (Kantar et al., 2016, 2018; Lundgren and Des Marais, 2020; Mora et al., 2018). Compared with annual species, perennials tend to be more stress-tolerant and require less irrigation and environmentally harmful inputs including pesticides and fertilizers (Glover et al., 2010). Nevertheless, previous attempts to develop a perennial crop through classical breeding methods has extended over long periods due to challenging crosses between 
species, a sever genetic drag, and declining yield under field conditions (Kantar et al., 2016; Lundgren and Des Marais, 2020). Therefore, breeding towards perennial crops should become more targeted and incorporate biotechnological practices in the breeding process (Hübner and Kantar, 2021).

To allow an efficient targeted breeding, perenniality has to be accurately defined. From a broad perspective, perenniality can be considered as a syndrome that is comprised of many interacting traits including growth rate, carbon fixation, root system development, source-sink dynamics (Lundgren and Des Marais, 2020), and involve different plant organs. In this study, we defined perennially as the ability to form a bulb organ in $H$. bulbosum and followed two approaches to explore the underlying molecular mechanism.

\section{Identifying perennial life habit genes}

The annual/perennial life habit has shifted in both directions along the evolution of many plant species (Heidel et al., 2016; Lundgren et al., 2020). This shift can be facilitated by a number of genetic mechanisms including gain or loss of "perenniality genes", changes in the regulation of gene expression, or critical change in protein structure (Heidel et al., 2016). Along the evolutionary history of the Hordeum genus, perennial and annual life habits have shifted number of times independently (Blattner, 2009), and the course of life habit evolution among $H$. bulbosum and $H$. spontaneum remains elusive. Thus, perenniality may have evolved independently in $H$. bulbosum through a process of gene gain or loss. To test this, a presence/absence variation (PAV) analysis was conducted between $H$. bulbosum and other annual cereal species and indicated that many disease responsive and development genes evolved uniquely in $H$. bulbosum (Figure 2B). Interestingly, the gene 14-3-3-like was detected only in $\mathrm{H}$. bulbosum and was previously found to promote storage organ formation in potato (Teo et al., 2017; Hannapel et al., 2017). This was further supported in the differential expression analysis, where 14-3-3-like was differentially expressed in response to the transition to LD regime in $\mathrm{H}$. bulbosum but not in $\mathrm{H}$. spontaneum. 


\section{Decoupling the genetic mechanism involved in flowering and bulb formation}

Another potential route for a perennial life habit to evolve is through modifications in genes expression that promote bulb formation and development. In $\mathrm{H}$. bulbosum, bulb formation is induced by a shift in day-length similarly to onion and potato (Lee et al., 2013; Zhang et al., 2020), and develops from the bottom of the shoot meristem (Leshem, 1971). Unlike onion and potato where transition to flowering inhibits the development of the storage organ, in $\mathrm{H}$. bulbosum flowering and bulb formation are coupled and occur simultaneously. To deconvolute bulb formation and flowering, a differential expression (DE) analysis was conducted using meristem tissues extracted before and after transition to long-day (LD) regime. The experiment was conducted in $H$. bulbosum and was compared with $H$. spontaneum where bulb initiating genes were not expected to be detected.

Comparison between the genes identified in $\mathrm{H}$. bulbosum and known regulation pathways of flowering and storage organ development in potato, onion and Arabidopsis indicated that the mechanism is largely conserved. Generally, the initiation of flowering and bulb formation begins with reception of light signal in the leaf tissue and transmitted to the shoot meristem through flowering locus T (Hannapel et al., 2017; Lee et al., 2013). Here, the experiment was conducted only with shoot meristems, thus FT was not detected in $\mathrm{H}$. bulbosum nor in $\mathrm{H}$. spontaneum. Among the candidate genes identified in $\mathrm{H}$. bulbosum, CO was upregulated and GIGANTEA was downregulated (Table S8). This expression profile promotes flowering in Arabidopsis (Putterill et al., 1995) but suppresses tuberization in potato (Abelenda et al., 2016, Hannapel et al., 2017). Moreover, the morphogenesis regulating gene COP9 which induces organ development (Chamovitz et al., 1996; Wei et al., 2003) was upregulated and so were several sugar transporters and starch biosynthesis genes including SWEET17 (Chardon et al., 2013).

This work sets the first milestone towards understanding the molecular mechanism of perenniality in $\mathrm{H}$. bulbosum. Therefore, we cautiously suggest a general scaffold for the 
molecular mechanism that underlies the development of a bulb organ (Figure S4). The activity of COP9 triggers a rapid cells division followed by sugar biosynthesis and accumulation of starch (MADS-box, Cell division control protein 48, SWEET17 and Phosphoglycerate mutase-like protein 4). Most of the pathway seem to be conserved among $H$. bulbosum and $H$. spontaneum, thus breeding towards a perennial crop may be more achievable than expected.

\section{Conclusions}

In this study, we assembled a reference transcriptome for $H$. bulbosum as a complementary infrastructure for the available draft genome. This platform has several advantages especially for targeting specific genes that are involved in bulb initiation and development. The PAV analysis of assembled transcripts and the differential expression analysis allowed to established the first milestone towards elucidating the molecular mechanism of bulb initiation and perenniality as was defined in $H$. bulbosum. The detected candidate genes should be further explored using genetic engineering techniques and later could potentially be applied in breeding programs to develop new perennial crop plants.

\section{Acknowledgements}

This work was supported by the German-Israeli Foundation (GIF), Grant: No.I-2501204.12-2018 


\section{References}

Abelenda JA, Cruz-Oró E, Franco-Zorrilla JM, Prat S. 2016. Potato StCONSTANS-like1 suppresses storage organ formation by directly activating the FT-like StSP5G repressor. Current Biology 26, 872-881.

Alexa A, Rahnenführer J, Lengauer T. 2006. Improved scoring of functional groups from gene expression data by decorrelating GO graph structure. Bioinformatics 22, 1600 1607.

Amstrong JM. 1936. Hybridization of triticum and agropyron: i. crossing results and description of the first generation hybrids. Canadian Journal of Research 14c, 190-202.

Andrews S. 2010. FastQC A Quality Control tool for High Throughput Sequence Data. Appels R, Eversole K, Feuillet C, et al. 2018. Shifting the limits in wheat research and breeding using a fully annotated reference genome. Science 361.

Blattner FR. 2009. Progress in phylogenetic analysis and a new infrageneric classification of the barley genus Hordeum (Poaceae: Triticeae). Breeding Science 59, 471-480.

Boeckmann B, Blatter MC, Famiglietti L, Hinz U, Lane L, Roechert B, Bairoch A. 2005. Protein variety and functional diversity: Swiss-Prot annotation in its biological context. Comptes Rendus - Biologies 328, 882-899.

Bolger AM, Lohse M, Usadel B. 2014. Trimmomatic: A flexible trimmer for Illumina sequence data. Bioinformatics 30, 2114-2120.

Bryant DM, Johnson K, DiTommaso T, et al. 2017. A Tissue-mapped axolotl de novo transcriptome enables identification of limb regeneration factors. Cell Reports 18, 762776.

Chamovitz DA, Wei N, Osterlund MT, Von Arnim AG, Staub JM, Matsui M, Deng XW. 1996. The COP9 complex, a novel multisubunit nuclear regulator involved in light control of a plant developmental switch. Cell 86, 115-121.

Chardon F, Bedu M, Calenge F, et al. 2013. Leaf fructose content is controlled by the 
vacuolar transporter SWEET17 in Arabidopsis. Current Biology 23, 697-702.

Consortium TU. 2021. UniProt: the universal protein knowledgebase in 2021. Nucleic Acids Research 49, D480-D489.

Costa-Silva J, Domingues D, Lopes FM. 2017. RNA-Seq differential expression analysis: an extended review and a software tool. PLOS ONE 12, 1-18.

Cui L, Ren Y, Murray TD, Yan W, Guo Q, Niu Y, Sun Y, Li H. 2018. Development of perennial wheat through hybridization between wheat and wheatgrasses: a review. Engineering 4, 507-513.

Eddy SR. 1998. Profile hidden Markov models. Bioinformatics 14, 755-763.

Finn RD, Bateman A, Clements J, et al. 2014. Pfam: the protein families database. Nucleic Acids Research 42, D222-D230.

Gasparini K, Moreira J dos R, Peres LEP, Zsögön A. 2021. De novo domestication of wild species to create crops with increased resilience and nutritional value. Current Opinion in Plant Biology 60, 1-7.

Grabherr MG, Haas BJ, Yassour M, et al. 2011. Full-length transcriptome assembly from RNA-Seq data without a reference genome. Nature Biotechnology 29, 644-652.

Haberer G, Mayer KFX. 2015. Barley: From Brittle to Stable Harvest. Cell 162, 469-471. Hannapel DJ, Sharma P, Lin T, Banerjee AK. 2017. The multiple signals that control tuber formation. Plant physiology 174, 845-856.

Heidel AJ, Kiefer C, Coupland G, Rose LE. 2016. Pinpointing genes underlying annual/perennial transitions with comparative genomics. BMC Genomics 17, 1-9.

Hübner S, Kantar MB. 2021. Tapping diversity from the wild: from sampling to implementation. Frontiers in Plant Science 12, 1-14.

J. D. Glover, J. P. Reganold, L. W. Bell, J. Borevitz, E. C. Brummer, E. S. Buckler, C. M. Cox, T. S. Cox, T. E. Crews, S. W. Culman, L. R. DeHaan, D. Eriksson, B. S. Gill, J. Holland, F. Hu, 
B. S. Hulke, A. M. H. Ibrahim, W. Jackson, S.S. Jones, S.C. Mur YX. 2010. Increased food and ecosystem security via perennial grains. Science 328, 1638-1639.

Jayakodi M, Padmarasu S, Haberer G, et al. 2020. The barley pan-genome reveals the hidden legacy of mutation breeding. Nature 588, 284-289.

Kantar MB, Hüber S, Herman A, et al. 2018. Neo-domestication of an interspecific tetraploid Helianthus annuus $\times$ Helianthus tuberous population that segregates for perennial habit. Genes 9, 1-13.

Kantar MB, Tyl CE, Dorn KM, et al. 2016. Perennial Grain and Oilseed Crops. Annual Review of Plant Biology 67, 703-729.

Koller D, Highkin HR. 1960. Environmental Control of Reproductive Development in Hordeum Bulbosum, a Perennial Pasture Grass. American Journal of Botany 47, 843847.

Langmead B, Trapnell C, Pop M, Salzberg SL. 2009. Ultrafast and memory-efficient alignment of short DNA sequences to the human genome. Genome Biology 10.

Larkin PJ, Newell MT, Hayes RC, Aktar J, Norton MR, Moroni SJ, Wade LJ. 2014. Progress in developing perennial wheats for grain and grazing. Crop and Pasture Science 65, 1147-1164.

Lee R, Baldwin S, Kenel F, McCallum J, Macknight R. 2013. FLOWERING LOCUS T genes control onion bulb formation and flowering. Nature Communications 4, 1-9.

Leshem B. 1971. Bulb Histogenesis in Hordeum bulbosum L. Annals of Botany 35, 57-62. Li B, Dewey CN. 2011. RSEM: accurate transcript quantification from RNA-Seq data with or without a reference genome. BMC Bioinformatics 12, 323.

Li H, Handsaker B, Wysoker A, Fennell T, Ruan J, Homer N, Marth G, Abecasis G, Durbin R. 2009. The Sequence Alignment/Map format and SAMtools. Bioinformatics 25, 20782079. 
Li B, Ruotti V, Stewart RM, Thomson JA, Dewey CN. 2010. RNA-Seq gene expression estimation with read mapping uncertainty. Bioinformatics 26, 493-500.

Love MI, Huber W, Anders S. 2014. Moderated estimation of fold change and dispersion for RNA-seq data with DESeq2. Genome Biology 15, 1-21.

Lundgren MR, Des Marais DL. 2020. Life history variation as a model for understanding trade-offs in plant-environment interactions. Current Biology 30, R180-R189.

Mora C, Spirandelli D, Franklin EC, et al. 2018. Broad threat to humanity from cumulative climate hazards intensified by greenhouse gas emissions. Nature Climate Change 8, 1062-1071.

Ofir M, Koller D. 1974. relationship between thermoinduction and photoinduction of flowering and dormancy in hordeum bulbosum L., a perennial grass. Functional Plant Biology 1, 259-270.

Petersen TN, Brunak S, von Heijne G, Nielsen H. 2011. SignalP 4.0: discriminating signal peptides from transmembrane regions. Nature Methods 8, 785-786.

Putterill J, Robson F, Lee K, Simon R, Coupland G. 1995. The CONSTANS gene of arabidopsis promotes flowering and encodes a protein showing similarities to zinc finger transcription factors. Cell 80, 847-857.

Ryan MR, Crews TE, Culman SW, DeHaan LR, Hayes RC, Jungers JM, Bakker MG. 2018. Managing for multifunctionality in perennial grain crops. BioScience 68, 294-304.

Shaar-Moshe L, Hübner S, Peleg Z. 2015. Identification of conserved drought-adaptive genes using a cross-species meta-analysis approach. BMC Plant Biology 15, 111.

Simão FA, Waterhouse RM, loannidis P, Kriventseva E V., Zdobnov EM. 2015. BUSCO: Assessing genome assembly and annotation completeness with single-copy orthologs. Bioinformatics 31, 3210-3212.

Teo CJ, Takahashi K, Shimizu K, Shimamoto K, Taoka KI. 2017. Potato tuber induction is regulated by interactions between components of a tuberigen complex. Plant and Cell 
Physiology 58, 365-374.

Vogel JP, Garvin DF, Mockler TC, et al. 2010. Genome sequencing and analysis of the model grass Brachypodium distachyon. Nature 463, 763-768.

Wang W, Mauleon R, Hu Z, et al. 2018. Genomic variation in 3,010 diverse accessions of Asian cultivated rice. Nature 557, 43-49.

Wei N, Deng XW. 2003. The COP9 Signalosome. Annual Review of Cell and Developmental Biology 19, 261-286.

Wendler N, Mascher M, Himmelbach A, Bini F, Kumlehn J, Stein N. 2017. A high-density, sequence-enriched genetic map of Hordeum bulbosum and Its Collinearity to H. vulgare. The Plant Genome 10, plantgenome2017.06.0049.

Yu H, Lin T, Meng X, et al. 2021. A route to de novo domestication of wild allotetraploid rice. Cell 184, 1156-1170.

Zhang X, Campbell R, Ducreux LM, et al. 2020. TERMINAL FLOWER-1/CENTRORADIALIS inhibits tuberisation via protein interaction with the tuberigen activation complex. Plant Journal 103, 2263-2278.

Zhong W, Gerstein M, Snydr M. 2009. RNA-Seq : a revolutionary tool for transcriptomics. Nature Reviews Genetics 10, 1-8. 